\title{
Building an Event Horizon Telescope: (sub)mm VLBI in the ALMA era
}

\section{Shep Doeleman*}

MIT Haystack Observatory

E-mail: sdoeleman@haystack.mit.edu

Recent technical and scientific progress in (sub)mm VLBI now makes it very likely that we will be able to observe and resolve a black hole event horizon within this decade. Over the next few years, new facilities, including ALMA, will join current (sub)mm VLBI arrays, creating a high sensitivity 'Event Horizon Telescope' (EHT) capable of angular resolutions approaching $20 \mu$ as. For SgrA*, the super massive black hole candidate at the Galactic Center, and M87, the black-hole powered core of a giant elliptical galaxy, these angular resolutions correspond to just a few Schwarzschild radii. Recent detections of both these sources confirm the existence of structure on event horizon scales. Enhancing the current (sub)mm VLBI array to enable imaging of these objects and time resolution of flaring structures will require technical development, but the needed improvements are relatively straightforward, making this a very tractable project. The work includes continued improvement in the bandwidth of VLBI backend systems, development of phased array systems to combine the collecting area of connected element arrays, deployment of modern ALMA-quality receivers, development of new (sub)mm VLBI sites, and migration to wideband software correlators.

10th European VLBI Network Symposium and EVN Users Meeting: VLBI and the new generation of radio arrays

September 20-24, 2010

Manchester Uk

\footnotetext{
* Speaker.
} 


\section{Approaching the Event Horizon}

New technical advances are leading to extension of Very Long Baseline interferometry to frontier wavelengths of $1.3 \mathrm{~mm}$ and $0.8 \mathrm{~mm}$. The major goal is to realize observations with angular resolutions approaching 20 micro arcseconds, but other important considerations include the ability to observe in a frequency range where the effects of interstellar scattering and faraday rotation (both with $\lambda^{2}$ dependencies) are greatly reduced. Many AGN also exhibit a spectral turnover in the submm range, suggesting that VLBI at high frequencies can be used to study sources in exceptional detail as they transition to the optically thin regime. For a few nearby AGN, (sub)mmVLBI can directly observe the immediate environment of the central super massive black hole (SMBH) with angular resolution comparable to the event horizon. Realizing this goal would open a new window on the study of General Relativity (GR) in the strong field regime, accretion and outflow processes at the edge of a black hole, the existence of event horizons, and fundamental black hole physics. Recent technical advances in (sub)mm VLBI coupled with new scientific observations have now brought such ultra-high resolution studies of black holes within reach.

The most compelling evidence for this is the $1.3 \mathrm{~mm}$ VLBI detection of Schwarzschild radius scale structure in $\mathrm{SgrA}^{*}$, the compact source of radio, submm, NIR and Xrays at the center of the Milky Way [1]. SgrA* is thought to mark the position of a $\sim 4 \times 10^{6} M_{\odot}$ black hole, which presents the largest apparent event horizon size of any black hole candidate in the Universe. This new 1.3 mm VLBI detection confirms that short wavelength VLBI of SgrA* can be used to directly probe the Event Horizon of this black hole candidate. More recent $1.3 \mathrm{~mm}$ VLBI detections of M87 (Virgo A) now confirm that a much more massive and luminous AGN can also be studied on Schwarzschild radius scales

\section{Building an Event Horizon Telescope}

The VLBI array used to detect Rsch scale structure in SgrA* and M87 consists of the ARO/SMT on Mt. Graham, AZ, two dishes of the CARMA array near Big Pine, CA, and the JCMT on Mauna Kea in Hawaii. The technical plan to transform this 3-site array into the high sensitivity, manybaseline EHT was submitted last year to the Radio/mm/submm (RMS) panel of the ASTRO2010 Decadal Review. The first phase focuses on achieving a 6-station array with a $4 \mathrm{GHz}$ bandwidth at an observing frequency of $230 \mathrm{GHz}$ within 4 years. The elements of this roadmap, and associated progress to date, are:

- Development and procurement of ultra-stable VLBI frequency references for $230 \mathrm{GHz}$ and $350 \mathrm{GHz}$ observations. Several of the Hydrogen maser frequency standards currently in use in the (sub)mm-VLBI array are vintage models, which difficult to maintain, and modern masers with excellent stability $\left(\sigma_{y}(1 s) \simeq 8 \times 10^{-14}, \sigma_{y}(10 s) \simeq 1.1 \times 10^{-14}\right)$ over 1-10second integration times (matching the coherence time of the atmosphere) can be purchased to replace them and outfit new sites. At the highest frequencies, sapphire oscillator based systems have even better stability and may be viable alternatives to Hydrogen masers standards.

- Continued development of VLBI backend instrumentation to increase recorded bandwidth. At present, the (sub)mm VLBI array records an aggregate data rate of $4 \mathrm{~Gb} / \mathrm{s}(1 \mathrm{GHz} \mathrm{BW}$ with 
2-bit sampling). ALMA-style receivers have an IF that is $8 \mathrm{GHz}$ wide with dual polarization, corresponding to $64 \mathrm{~Gb} / \mathrm{s}$. By harnessing commercial hardware trends, new VLBI systems that can record at these speeds will be brought on line within a few years. Such a bandwidth increase will boost array sensitivities by a factor of $\times 4$.

- Adding new sites to the array. The current three site array will soon be joined by other (sub)mm VLBI facilities including the IRAM 30m and Plateau de Bure array, both of which have carried out successful $1.3 \mathrm{~mm}$ VLBI in the past [2]. In addition, the LMT facility, under construction in Mexico, will significantly improve baseline coverage and sensitivity of the array. New telescopes in Chile, near the ALMA site, are due to come on line in 2011, and these sites (the APEX and ASTE telescopes) will improve angular resolution of the EHT to $\sim 35 \mu$ as at $1.3 \mathrm{~mm}$ wavelength. By moving to $0.8 \mathrm{~mm}$ wavelength, the angular resolution on the longest baselines reaches $\sim 22 \mu$ as. These sites, either in operation or with current plans to join the array, are shown in red on the globes in Figure 1. Additional telescopes that may join the array in the future include SEST in Chile, the South Pole Telescope, and the Haystack $37 \mathrm{~m}$ telescope (currently undergoing a high frequency surface upgrade), and are shown in yellow (Fig. 1). Other potential sites that are being explored include sites in New Zealand, and two in Africa (shown in green). Imaging simulations of theoretical SgrA* models using sub-arrays of these sites are shown in Figure 2
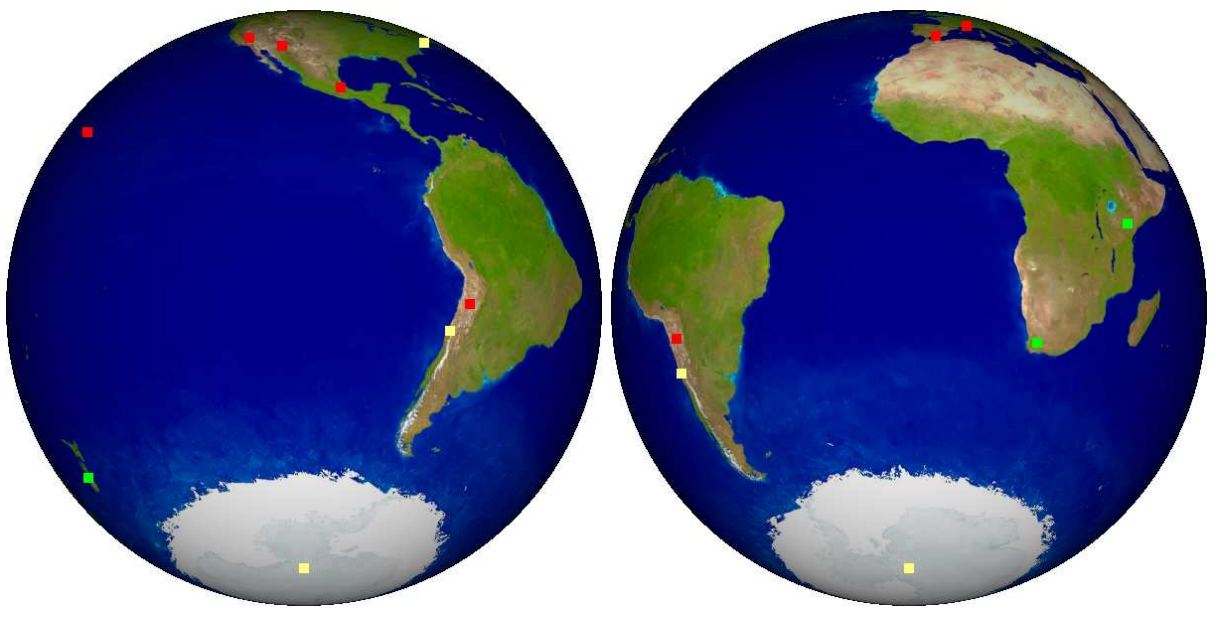

Figure 1: Locations of current, planned, and potential EHT sites.

- Phasing connected element interferometers into single VLBI elements. Several of the current or planned EHT sites are (sub)mm connected element arrays, whose elements can be coherently combined into a single effective aperture for VLBI. A phased array processor to combined the Caltech Submillimeter Observatory, the James Clerk Maxwell Telescope, and the Submillimeter Array dishes on Mauna Kea has been designed, tested and deployed for VLBI observations [3]. Integration of this system at the CARMA site will allow up to six of the 10m CARMA dishes to be coherently added by April 2011, for an increase in sensitivity on the CARMA-Hawaii baseline of $\sim \times 4-5$. Of primary importance to the EHT will be the inclusion of ALMA. When all $\sim 5012 \mathrm{~m}$ dishes of ALMA are phased together as a single 
VLBI element, baselines from ALMA to Hawaii will detect SgrA* with a signal to noise of $\sim 20$ in 10 seconds, assuming 4GHz BW. This will allow VLBI to monitor time-variable structures in the accretion flow near the black hole using non-imaging techniques (Figure 3, [4]).

- A wideband VLBI correlator for (sub)mm work. The correlator functions as the "lens" in VLBI, cross-correlating signals from all sites to produce Fourier components, which are then used to model or image objects on the sky. The EHT places severe requirements on data rate and upper frequency range in the correlator, and use of existing hardware based processors for future EHT work will not be feasible. In addition, current hardware correlators cannot accommodate the unique data output expected from ALMA when it joins the EHT array. Planned efforts will migrate the processing of (sub)mm VLBI data to a softwarebased correlator system (the well-tested DiFX algorithm: [5]), which has the flexibility and computational power to accommodate new EHT requirements while preserving a sustainable expansion path to higher data rates

- Building state-of-the-art (sub)mm Receivers for the EHT. Several of the current (sub)mm VLBI sites use older $1.3 \mathrm{~mm}$ and $0.8 \mathrm{~mm}$ receivers, with Single Sideband (SSB) receiver noise temperatures more than double that of modern systems, without dual-polarization capability, and with narrow IF bandwidths. Within the international ALMA program, a large receiver development project has designed, tested, and fabricated natively SSB mixers and preamps that set performance standards in the 1.3 and $0.8 \mathrm{~mm}$ bands. Work is planned to leverage this Globally supported receiver development to build new receivers for select EHT sites. At the JCMT, for example, straightforward integration of existing 1.3mm ALMA mixer/preamp modules will result in dual-polarization capability, $\mathrm{x} 2.5$ reduction in receiver noise, and IF bandwidth increases to $8 \mathrm{GHz}$.

\section{A Broad VLBI Science Case}

In addition to science that focuses on the Event Horizon (through observations of SgrA*, M87, and potentially CenA), the EHT will provide unsurpassed angular resolution for more general studies of relativistic jets. These include linking flaring activity in other wavebands (xray and gamma rays) to the emergence of VLBI jet components (e.g. [6]), and using transverse jet structure including polarization for comparison with jet collimation and propagation theory [7]. Extrapolating from the 3.5mm VLBI survey of Lee et al (2008), one estimates that there are >50 AGN sources detectable with the $1.3 \mathrm{~mm}$ EHT at a signal to noise ratio (per baseline) of $>5$ in 10 seconds. At lower frequencies, the inclusion of a phased ALMA in VLBA observations at $7 \mathrm{~mm}$ and $3 \mathrm{~mm}$ will more than double the angular resolution of the VLBA and provide exceptionally sensitive new North-South baselines (the total aggregate collecting area of ALMA exceeds the collecting area of the VLBA).

The EHT will also open up a new and rich frequency region for astronomical maser studies. A wide variety of $\mathrm{mm}$ and submm maser species can be observed with high angular resolution including water, SiO, HCN, Methanol, and Hydrogen Recombination Lines [9]. These masers 

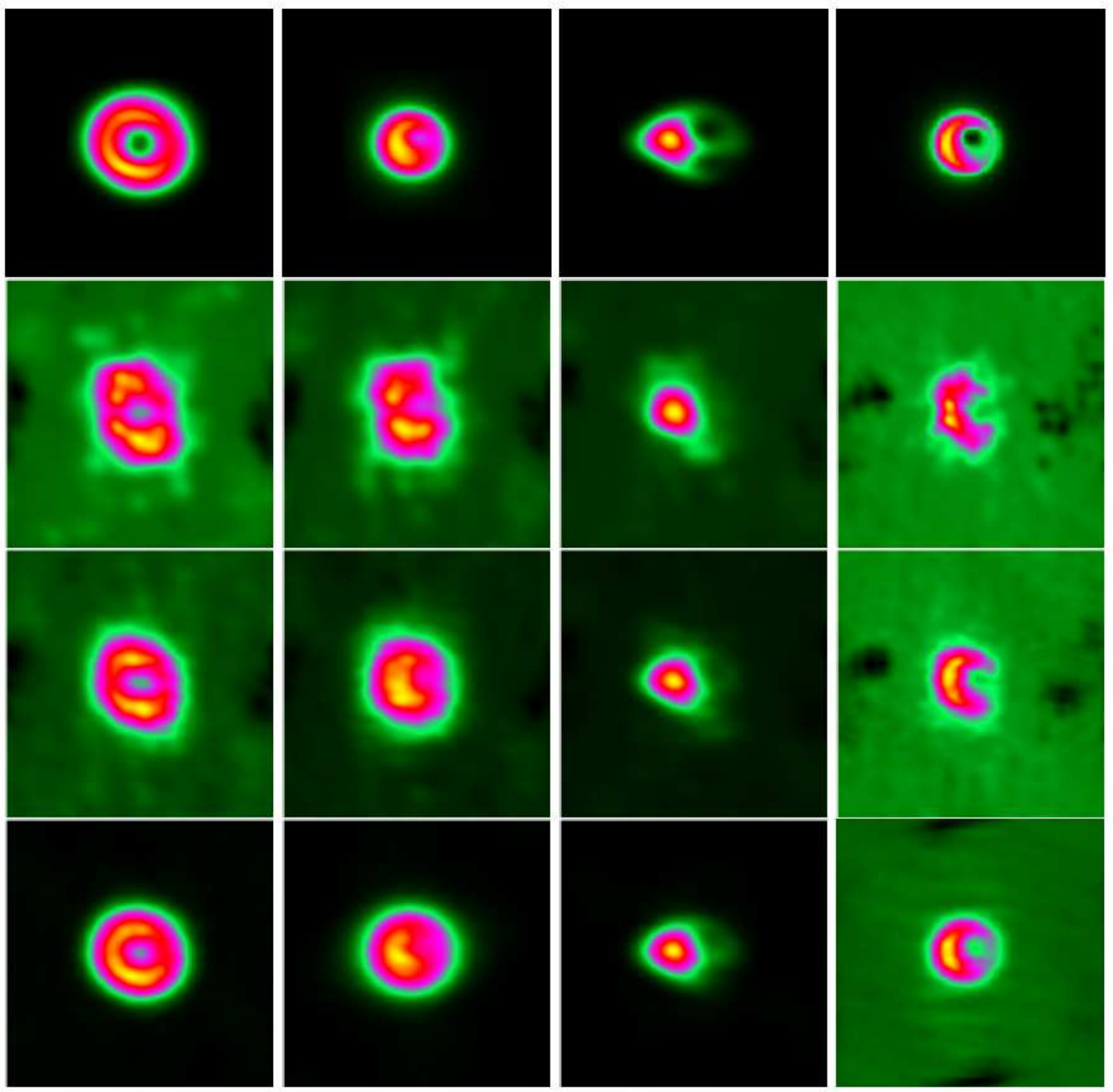

Figure 2: Simulations and imaging reconstructions of Schwarzschild radius scale structures surrounding the Event Horizon of the $\sim 4 \times 10^{6} M_{\odot}$ black hole at the Galactic Center. The top row shows emission models that have been convolved with ISM wavelength dependent scattering effects. The four models are (from the left): [230GHz GRMHD model, BH spin a=0.9, accretion disk face on], [230 GHz Radiatively Inefficient Accretion Flow (RIAF) model, BH spin a=0, accretion disk 30deg inclination], [230GHz GRMHD model, BH spin a=0.9, accretion disk 80deg inclination], [345GHz RIAF model, BH spin a=0, accretion disk 30deg inclination]. The second, third and fourth rows show imaging results with the following (in order) arrays: Phase I (CARMA, Hawaii, SMTO, PdeBure, IRAM30m, Chile, LMT), Phase I + S. Pole + S. Africa, Phase III (all 13 potential sites). Models courtesy J. Dexter (GRMHD) and Avery Broderick (RIAF). Each image is $\pm 138 \mu$ as on a side (about \pm 14 Rsch). 

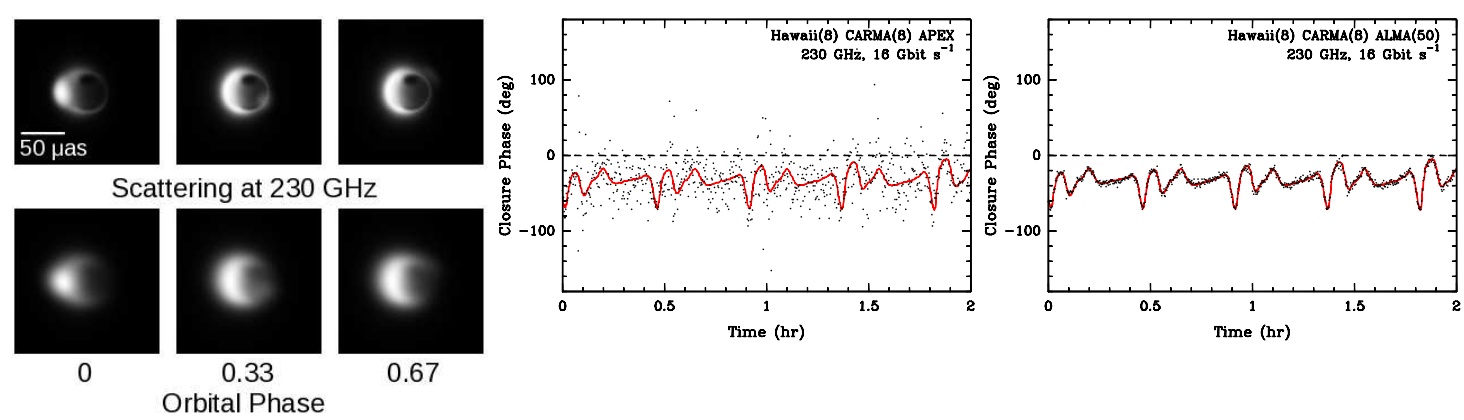

Figure 3: Non-imaging signature of a hot-spot orbiting a spin zero black hole at a radius of $3 R_{\text {sch }}$ with a period of 27 minutes. Left panel shows the model, without ISM scattering above and with ISM scattering below, for three phases in the hot-spot orbit. Right panels show the expected closure phase (a quantity derived from phases on a closed triangle of 3 VLBI baselines) from the model (red) and simulated 10 second closure phase points on a $1.3 \mathrm{~mm}$ VLBI array consisting of Hawaii, CARMA, and either 1 or 50 phased dishes of ALMA at $16 \mathrm{Gbit} s^{-1}$ recording rate. With one dish in Chile the closure phases average to less than zero, implying asymmetric structure (middle panel). But the combination of wider bandwidth, and phasing 50 ALMA dishes enables detailed monitoring of orbiting structures in the accretion flow (right panel) [4].

occur in a wide range of astronomical contexts, from star forming regions to the circumstellar envelopes of evolved stars.

High frequency VLBI work at Haystack Observatory is supported by NSF grants AST-0908731, AST-0905844, AST-0807843.

\section{References}

[1] S. S. Doeleman et al. 2008, Event-horizon-scale structure in the supermassive black hole candidate at the Galactic Centre, Nature $\mathbf{4 5 5} 78$.

[2] Krichbaum, T. P., et al. 1998, VLBI observations of the galactic center source SGR $A^{*}$ at $86 G H z$ and $215 \mathrm{GHz}, A \& A 335 \mathrm{~L} 106$.

[3] Weintroub, J. 2008, A Submillimeter VLBI Array, Journal of Physics: Conference Series 131012047.

[4] S. S. Doeleman, V. L. Fish, A. E. Broderick, A. Loeb, \& A. E. E. Rogers 2009, Detecting Flaring Structures in Sagittarius A* with High-Frequency VLBI, ApJ 69559.

[5] Deller, A. T., Tingay, S. J., Bailes, M. \& West, C. 2007, DiFX: A Software Correlator for Very Long Baseline Interferometry Using Multiprocessor Computing Environments, PASP 119318.

[6] Marscher, A. P., et. al. 2010, Probing the Inner Jet of the Quasar PKS 1510-089 with Multi-Waveband Monitoring During Strong Gamma-Ray Activity, ApJL 710 L126.

[7] Piner, B. G., Pant, N. \& Edwards, P. G. 2010, The Jets of TeV Blazars at Higher Resolution: $43 \mathrm{GHz}$ and Polarimetric VLBA Observations from 2005 to 2009, ApJ $\mathbf{7 2 3} 1150$.

[8] Lee, S., et al. 2008 A Global 86GHz VLBI Survey of Compact Radio Sources, AJ 136159.

[9] Humphreys, E. M. L. 2008 Submillimeter and millimeter masers, Proceedings IAU Symposium 242 471. 